Onomástica Desde América Latina, n.3, v.2, janeiro - junho, 2021, p. 2 - 29. ISSN 2675-2719

https://doi.org/10.48075/odal.v0i0.25781

\title{
Tendencies of formation of anthroponyms of rural residents in the oldest Lithuanian parish register of Joniškis dated 1599 - 1621
}

\section{Tendências de formação de antropônimos de residentes rurais nos registros de 1599 - 1621 da paróquia mais antiga da Lituânia}

\author{
Alma Ragauskaitè \\ Institute of the Lithuanian Language \\ https://orcid.org/0000-0001-5952-8027 \\ alma.ragauskaite@1ki.lt
}

\begin{abstract}
Parish registers are among the most important sources of Lithuanian historical anthroponymy. A chronologically unique baptismal register of Joniškis dating to 1599-1621, which is currently the oldest known Lithuanian parish register in Lithuania, stands out in the context of such registers. This article delved into the oldest inscriptions dated 1599-1600, which were found in the parish register of Joniškis, describes and analyses 290 personal names (second members of the binary naming model) in terms of formation. The following major research methods are used to discuss the data of historical anthroponymy: descriptive and comparative. The 290 analysed personal names were classified into two groups: anthroponyms with patronymic suffixes and anthroponyms without such suffixes. The first group was much larger. It comprised 274 (or 94.5\%) of all anthroponyms. The second group had $16(5.5 \%)$ personal names.
\end{abstract}

Keywords: Joniškis; baptismal register; historical anthroponymy; Lithuanian historical anthroponym; word-formation.

\section{Resumo}

Os registros paroquiais estão entre as fontes mais importantes da antroponímia histórica lituana. Trata-se de certidões de batismo cronologicamente únicas de Joniškis datados de 1599-1621, que são, atualmente, o mais antigo registro paroquial lituano conhecido na Lituânia e que se destaca no contexto de tais registros. Este artigo aprofundou-se nas inscrições mais antigas datadas de 1599-1600, que foram encontradas no registro paroquial de Joniškis, descreve e analisa 290 nomes pessoais (segundo membro do modelo binário de nomeação) em termos de formação. Para tanto, foram utilizados, principalmente, os métodos de pesquisa descritivo e comparativos de antroponímia histórica. Os primeiros membros das instâncias de inscrição binária eram nomes cristãos. Os 290 nomes pessoais analisados foram classificados em dois grupos: antropônimos com sufixos patronímicos e antroponímicos sem tais sufixos. O primeiro grupo é muito maior que o segundo: enquanto, no primeiro, há 274 nomes (ou seja 94,5\%) de todos os segundos antropônimos, no segundo, há apenas 16 nomes (ou seja 5,5\%).

Palavras-chave: Joniškis; certidões de batismo; antroponímia histórica; antroponímia histórica lituana; formação de palavras. 
Onomástica Desde América Latina, n.3, v.2, janeiro - junho, 2021, p. 2 - 29. ISSN 2675-2719

https://doi.org/10.48075/odal.v0i0.25781

Studies have already commenced on the old anthroponymy of Joniškis ${ }^{1}$. First publications focused on male forms of anthroponyms with Lithuanian formants from the oldest Lithuanian parish register of baptism of Joniškis dated 1599-1621 (GARLIAUSKAS, 1998: 152-153). Analysis into the origins of the same-period personal names particular to Joniškis residents (males) determined that nicknames were the most frequent source ${ }^{2}$ among reconstructed anthroponyms of Lithuanian origin while various forms of Christian names and their suffixal derivatives were the most likely source for the reconstructed personal names that originated from baptismal names ${ }^{3}$ (RAGAUSKAITE், 2004: 16-17). A separate focus was placed on the functioning of Lithuanian suffixes in personal names of Joniškis residents (females) of the end of the 16 th - the beginning of the 17th centuries ${ }^{4}$ (RAGAUSKAITE், 2015: 55-74). Based on the analysis into origins of binary naming cases of villagers available in the parish register of baptism of Joniškis dated 1599_ 1621, Lithuanian derivation was established for the total of 187 anthroponyms. Out of 187 discussed personal names of Lithuanian origin, 112 different anthroponym forms were reconstructed. Among them are 36 (32\%) Lithuanian old two-stem anthroponyms, 19 (17\%) abbreviations of two-stem personal names and their suffixal derivatives and 57 (51 \%) anthroponyms of nickname origin ${ }^{5}$ (RAGAUSKAITE், 2019b: 162).

Considering the peculiarities specific to the naming of Joniškis residents (male and female) as well as the origin of their personal names and other distinctions, further investigation into

\footnotetext{
${ }^{1}$ A description of Lithuanian personal name system can be found in English in: Ragauskaite (2008); Seide \& Petrulione (2018); in Spanish in Seide (2020) and in Portuguese in Amaral \& Seide (2020).

${ }^{2}$ Bakša, Burba, Dešrius, Gulbè, Gumbis, Kubilius, Laukis, Laumis, Miknius, Ožys, Rūguitis, Sliekuitis, Šukis, Trumpaitis, Žeimys.

${ }^{3}$ Banys, Bernotuitis, Čepas, Gabrytis, Juozuitis, Maculis, Mikutis, Mitkus, Povilas, Sabas, Stanys, Šimkus, Vaičiulis, Vaitkūnas, Venckūnas.

4 Bačkiūčia, Baskutaičia, Düdienè, Jereminaičia, Kizyčia, Luknaičia, Mažeikienè, Medginyčia, Mižutaitienè, Paruitienè, Siklyčia, Staliorienè, Stangaičia, Velikienè, Žirnyčia.

5 Budginis, Kybutaitis, Milvydaitis, Rimdauguitis, Visnoraitis; Beinius, Gineikaitis, Noreikaitis, Vaidotaitis, Žadeikuitis; Balčaitis, Diržaitis, Karaliūnas, Labutaitis, Šarkaitis.
} 
Onomástica Desde América Latina, n.3, v.2, janeiro - junho, 2021, p. 2 - 29. ISSN 2675-2719 https://doi.org/10.48075/odal.v0i0.25781

historical anthroponymy of the old parish villagers is relevant and should be continued. This article aims to investigate the oldest inscriptions dated 1599-1600 of the parish register of Joniškis, and analyse 290 personal names (second members of the binary naming model) from the perspective of formation. To pursuit those aims this article: 1) describe names (first members of the binary naming instances); 2) indicate the most productive suffixes of personal names; 3) recreate forms of anthroponyms with formants of Lithuanian formation and compare them with contemporary Lithuanian surnames ${ }^{6}$; 4 ) identify and count the historical personal names under investigation that are no longer functional in contemporary index of the surnames.

Anthroponymic material was collected from the old parish register of baptism of Joniškis dated $1599-1621^{7}$. The original title of the manuscript source is Liber Baptisatoru $[\mathrm{m}]^{8} \|$ Ecc[lesi] e Parochialis Jani $=\|$ scensis ab Anno 1599 . - \| usq[ue] ad An[n]um \| 1621. - . The fly leaf contains another title that reads CATA: \| LOGVS. \| BAPTISATO: || RVM. EC: \| CLESIEE. || IANI: || SCEN: || SIS. In 1594-1621, Benediktas Sviechovskis was the parson of the Joniškis Church of the Assumption of the Virgin Mary. As believed, he had initiated this chronologically unique source in 1599 (ALIŠAUSKAS, JASZCZOŁT, JOVAIŠA \& PAKNYS, 2009: 79). The manuscript was kept in Latin and Polish (GARLIAUSKAS, 2004: 21-22; RAGAUSKAITĖ, 2015: 58). The entire book contains consistent Latin inscriptions of years and month names. The

\footnotetext{
${ }^{6}$ The manuscript was written in Polish and Latin. Hence, the anthroponyms of Lithuanian settlers were Latinized, Slavicized and could be differently modified in different records of the baptismal register. Certain facts of Slavicization of personal names were distinguished in the historical documents under analysis: sound substitution, use of Slavic suffixes in the anthroponyms of Lithuanian origin, omission of endings, etc. Based on these principles, personal names of Joniškis parish villagers of 1599-1600 were analysed and reconstructed. For more details of this type of anthroponomastic research see MACIEJAUSKIENĖ, 1994: 25-30; RAGAUSKAITE், 1999: 148-149; 2005 : 61-66; 2015: 63-64; 2018b: 57-58; 2019a: 182-183.

${ }^{7}$ See the fragments of this archival source at the end of the article.

${ }^{8}$ Abbreviations used in the manuscript are supplemented. Additions are indicated in square brackets.
} 
Onomástica Desde América Latina, n.3, v.2, janeiro - junho, 2021, p. 2 - 29. ISSN 2675-2719

https://doi.org/10.48075/odal.v0i0.25781

comparison of handwriting reveals that the book was kept by several people. Formulas for the registration of baptism varied. Apparently, the text of inscriptions was often abbreviated.

\section{The patterns of naming system}

It is important to emphasise which personal names inscribed in church sources are attributable to villagers of the Joniškis parish (RAGAUSKAITE், 2000: 95-97; 2001: 124-125; 2002: 32-34; 2003: 83-85; 2006: 82-83; 2009: 227-228; 2014a: 8-9). Indications of villages in Latin and Polish help to determine the required anthroponyms, e.g., 1600 Bartholomaus Andresziuns <...> Pagi Girdziuna LBEPJ 89; 1599 Baniulis Andrieiuns de villa Girdziunu LBEPJ 4; 1600 Genis Banaitis <...> Pagi Eziekiow LBEPJ 16; 1599 Motieiaus Bartkuna de villa Walunu gen. sg. ${ }^{10}$ LBEPJ 4; 1600 Valentinus Bertaszaitis <...> Pagi Berzenu LBEPJ 10; 1600 Nicolaus Janufzaitis <...> pagi Krampiu LBEPJ 14; 1600 Josephus Jenaitis <...> Pagi Eziekia LBEPJ 19; 1600 Nicola[us] Jocubaitis <...> Pagi Girdziunow LBEPJ 16; 1600 Nicolaus Jukiszkaitis <...> pagi skakow LBEPJ 15; 1600 Bartholomaeus Jurgaitis <...> Pagi Szymkunu LBEPJ 14v; 1600 Mathaus Koczuitis <...> Pagi Normanciow LBEPJ 12; 1600 Matulis Eaucuns <...> Pagi Zadwayniu LBEPJ 17; 1600 Gregorius Łaurinaytis <...> Pagi Tauciunu LBEPJ 17; 1599 Jauniunu Aduocationis ${ }^{11}$ Ciapula Mantiurgaycia gen. sg. LBEPJ 2v; 1600 Mathous Petrosius <...> Pagi Proćiunow LBEPJ 8v; 1600 Laurentius Stanaytis <...> Pagi Selmekia LBEPJ 11v; 1599 Vrbons

\footnotetext{
${ }^{9}$ All historical personal names provided in the article are registered. Each indicates the year the anthroponym was recorded, the abbreviation of the historical source (LBEPJ) and the page number. Personal names were presented attempting to preserve their original forms recorded in parish register.

${ }^{10}$ See the list of abbreviations: comp. - compare; curt. - current; gen. - Lat. (cãsus) genetīvus "genitive case"; Lat. Latin; n. - name; sg. - Lat. (numerus) singulāris "singular"; sur. - surname.

${ }^{11}$ In some instances, this parish register indicates the vaitas (chief town administrator) of Šiauliai Volost (factual Šiauliai Crown Estate since 1616), where the village of Joniškis is located (ŽILYS, 2012: 127).
} 
Onomástica Desde América Latina, n.3, v.2, janeiro - junho, 2021, p. 2 - 29. ISSN 2675-2719

https://doi.org/10.48075/odal.v0i0.25781

Szariuitis de villa Wayniekiu LBEPJ 2v; 1599 Stanisława Zaragaicia <...> de villa Łakayciu gen. sg. LBEPJ 3.

The parish villages were populated with parents and godparents of christened babies, e.g., 1600 Patr[ini] Gabriel Augaitis <...> Pagi Wekoniow LBEPJ 9; 1600 Patr[ini] Mathaus Jukaitis <...> Pagi Kipsćia LBEPJ 8v; 1600 Patr[ini] Michael Kiemienciuns <...> Pagi Gudayciow LBEPJ 12v; 1600 Patr[ini] Gregorius Łaurinaytis <...> Pagi Tauciunu LBEPJ 17; 1600 Patr[ini] Jacobus Locaitis <...> pagi Donilu LBEPJ 14v; 1600 Patr[ini] Gregorius Makaweckuns <...> pagi Subaciunu LBEPJ 14v; 1600 Patr[ini] Nicolaus Mockaitis <...> Pagi Waydminia LBEPJ 17; 1600 Pagi Szłapakiu Patrini Macis Petraitis LBEPJ 6v; 1600 Patr[ini] Paulus Staniulis <...> Pagi Radikiu LBEPJ 9v; 1599 Compatres <...> Petru[m] Tautoßaitis <...> de uillâ Domeikiu LBEPJ 1; 1600 Patr[ini] Matulis Wiluns <...> Pagi bariuna LBEPJ 13v; 1600 Patr[ini] Vrbanus Zalwaruytis <...> Pagi Andrefiunu LBEPJ 17; 1600 Patr[ini] Stafius Zaruytis <...> Pagi Trapayciow LBEPJ 16.

Christian names are used at the first components of this type of naming. Their various forms are divided into three groups: 1) long canonical forms, 2) hypocoristics ${ }^{12}$ and 3) diminutive forms of the hypocoristics.

First members of binary naming instances were usually traditional Christian names, e.g., 1600 Adami gen. sg. LBEPJ 10; 1600 Adamus LBEPJ 16v - comp. curt. n. Adõmas (LVKŽ ${ }^{13}$ 61); 1600 Alberti gen. sg. LBEPJ 8v - comp. curt. n. Álbertas (LVKŽ 64); 1600 Ambrosius LBEPJ 16v

\footnotetext{
12 "A hypocoristic form can be understood as a unit formed from a morphological variation (clipping, diminutive, augmentative, etc.) of another anthroponym. It is usually used in familiar contexts. Hypocoristic forms differ from nicknames because they originate from a full form of another proper name" (SEIDE \& PETRULIONE 2020: 2).

${ }^{13}$ All current names provided in the article are registered (LVKŽ - Kuzavinis, K., \& Savukynas, B. (1994). Lietuviu vardu kilmés žodynas. Vilnius: Mokslo ir enciklopedijų leidykla).
} 
Onomástica Desde América Latina, n.3, v.2, janeiro - junho, 2021, p. 2 - 29. ISSN 2675-2719

https://doi.org/10.48075/odal.v0i0.25781

- comp. curt. n. Ambraziẽjus (LVKŽ 73); 1600 Andreas LBEPJ 8; 1599 Andrieiaus gen. sg. LBEPJ 4 - comp. curt. n. Andriẽjus (LVKŽ 74); 1600 Antonius LBEPJ 18 - comp. curt. n. Antãnas (LVKŽ 76); 1600 Augustini gen. sg. LBEPJ 14; 1600 Auguftinus LBEPJ 10v - comp. curt. n. Augustinas (LVKŽ 85); 1600 Balthoromaus LBEPJ 8v; 1600 Baltoromaus LBEPJ 10v; 1599 Battromieius LBEPJ 3v; 1600 Bartholomaus LBEPJ 7v; 1600 Bartholoma[us] LBEPJ 8 - comp. curt. $\mathrm{n}$. Baltramiẽjus (LVKŽ 89); 1599 Bernardi gen. sg. LBEPJ 3; 1599 Bernardus LBEPJ 1 - comp. curt. n. Bernárdas (LVKŽ 96); 1600 Gabriel LBEPJ 9 - comp. curt. n. Gabriẽlius (LVKŽ 160); 1600 Gaspar LBEPJ 11v; 1600 Gaspari gen. sg. LBEPJ 12 - comp. curt. n. Gãsparas (LVKŽ 164); 1599 Georgy LBEPJ 1; 1600 Georgius LBEPJ 8; 1600 Gregory LBEPJ 8; 1600 Gregorÿ LBEPJ 11; 1600 Gregorius LBEPJ 13; 1600 Jurgis LBEPJ 14 - comp. curt. n. Jùrgis (LVKŽ 215); 1600 Iacobi gen. sg. LBEPJ 15; 1600 Jacobi gen. sg. LBEPJ 2; 1600 Jacobus LBEPJ 9; 1600 Jacob[us] LBEPJ 11; 1599 Jokuba gen. sg. LBEPJ 3 - comp. curt. n. Jokūbas (LVKŽ 207); 1600 Jan LBEPJ 12v-13; 1599 Joannes LBEPJ 5; 1599 Jona gen. sg. LBEPJ 4 - comp. curt. n. Jõnas (LVKŽ 208); 1600 Josephi gen. sg. LBEPJ 9 - comp. curt. n. Juõzapas (LVKŽ 214); 1600 Laurenty LBEPJ 8v; 1600 Laurentÿ LBEPJ 13; 1600 Laurentius LBEPJ 11v - comp. curt. n. Laurỹnas (LVKŽ 242); 1600 Lucas LBEPJ 15 - comp. curt. n. Lùkas (LVKŽ 255); 1599 Eukosziaus gen. sg. LBEPJ 4 - comp. curt. n. Lukõšius (LVKŽ 255); 1600 Marcus LBEPJ 9v comp. curt. n. Mõrkus (LVKŽ 279); 1599 Martina gen. sg. LBEPJ 3v; 1600 Martini gen. sg. LBEPJ 9v - comp. curt. n. Martỹnas (LVKŽ 264); 1600 Mathæeus LBEPJ 8v; 1600 Mathæe[us] LBEPJ 17; 1600 Mathias LBEPJ 10v; 1599 Mothieiaus gen. sg. LBEPJ 3; 1599 Motieiaus gen. sg. LBEPJ 4 - comp. curt. n. Motiẽjus (LVKŽ 279); 1600 Michael LBEPJ 12v; 1599 Mikatoiaus gen. sg. LBEPJ 3v; 1600 Nicolaus LBEPJ 9v; 1600 Nicola[us] LBEPJ 16 - comp. curt. n. Mikalõjus (LVKŽ 270); 1599 Pauli gen. sg. LBEPJ 1v; 1600 Paulus LBEPJ 16v; 1600 Paul[us] 
Onomástica Desde América Latina, n.3, v.2, janeiro - junho, 2021, p. 2 - 29. ISSN 2675-2719 https://doi.org/10.48075/odal.v0i0.25781

LBEPJ 14 - comp. curt. n. Paũlius (LVKŽ 298); 1600 Petri gen. sg. LBEPJ 12; 1600 Petrus LBEPJ 10v - comp. curt. n. Pẽtras (LVKŽ 299); 1599 Powiła gen. sg. LBEPJ 4 - comp. curt. n. Póvilas (LVKŽ 301); 1600 Sebeftiani gen. sg. LBEPJ 10v - comp. curt. n. Sebastijõnas (LVKŽ 325); 1600 Simon LBEPJ 7v; 1600 Simonis gen. sg. LBEPJ 10v - comp. curt. n. Simonas (LVKŽ 329); 1600 Stanislaus LBEPJ 2; 1600 Stanisla[us] LBEPJ 14; 1599 Stanistawa gen. sg. LBEPJ 3 - comp. curt. n. Stanìslovas (LVKŽ 335); 1600 Stephani gen. sg. LBEPJ 11; 1600 Stephanus LBEPJ 8v; 1600 Sthephani gen. sg. LBEPJ 9; 1600 Sthephanus LBEPJ 6 - comp. curt. n. Stẽponas (LVKŽ 336); 1599 Toma LBEPJ 2 - comp. curt. n. Tòmas (LVKŽ 350); 1600 Vrbani gen. sg. LBEPJ 15; 1600 Vrbanus LBEPJ 30; 1599 vrbona gen. sg. LBEPJ 3v; 1599 Vrbons LBEPJ 2v comp. curt. n. Ùrbonas (LVKŽ 353-354); 1599 Waitiekaus gen. sg. LBEPJ 4 - comp. curt. n. Vaitiẽkus (LVKŽ 361); 1599 Valentini gen. sg. LBEPJ 1v; 1600 Valentinus LBEPJ 16v - comp. curt. n. Valentìnas (LVKŽ 363).

Only a few instances of canonical Christian names hypocoristics were found, e.g., 1600 Genis LBEPJ 16 - comp. curt. n. Gènis (LVKŽ 174) < Eugènijus (LVKŽ 153); 1600 Macis LBEPJ 6v - comp. curt. n. Macỹs (LVKŽ 280) < Motiẽjus (LVKŽ 279-280); 1599 Stas LBEPJ 4v; 1599 Stasiaus gen. sg. LBEPJ 3; 1600 Stafius LBEPJ 16 - comp. curt. n. Stasỹs, Stãsius (LVKŽ 335) < Stanislovas (LVKŽ 335). Second members of binary naming instances were personal names with or without suffixes, which is further analysed in terms of formation.

Fewer diminutive forms (with diminutive suffixes) of baptismal names hypocoristics were noticed, e.g., 1600 Antulis LBEPJ 15 - comp. curt. n. Ántas (LVKŽ 76) < Antãnas (LVKŽ 76); 1599 Baniulis LBEPJ 4 - comp. curt. n. Bãnis, Banỹs (LVKŽ 95) < Benedìktas (LVKŽ 95); 1600 Geniulis LBEPJ 21; 1600 Genulis LBEPJ 12 - comp. curt. n. Gènis (LVKŽ 174) < Eugènijus (LVKŽ 153); 1599 Jonela gen. sg. LBEPJ 4v; 1600 Jonelis LBEPJ 9v - comp. curt. n. Jõnas 
Onomástica Desde América Latina, n.3, v.2, janeiro - junho, 2021, p. 2 - 29. ISSN 2675-2719

https://doi.org/10.48075/odal.v0i0.25781

(LVKŽ 208); 1599 Jonula gen. sg. LBEPJ 4 - comp. curt. n. Jõnas (LVKŽ 208); 1600 Jurgelis

LBEPJ 12 - comp. curt. n. Jùrgis (LVKŽ 215); 1599 Matula gen. sg. LBEPJ 4; 1600 Matulis

LBEPJ 13v - comp. curt. n. Mãtas (LVKŽ 265) < Motiẽjus (LVKŽ 279-280); 1600 Staniulis

LBEPJ 13 - comp. curt. n. Stãnis, Stanỹs (LVKŽ 335) < Stanìslovas (LVKŽ 335); 1600 Valulis

LBEPJ 16v - comp. curt. n. Valỹs (LVKŽ 364) < Valentìnas (LVKŽ 363).

\section{Characteristics of anthroponym formation}

The origin and structure of a historical anthroponym is not necessarily indicative of its bearer's ethnicity (RAGAUSKAITĖ, 2014b: 325-327; 2016a: 46-49; 2016b: 112-115; 2017b: 5457; 2018c: 176; 2019b: 155). The 290 discussed personal names dated 1599-1600 were classified into two groups: 1) anthroponyms with patronymic suffixes, namely, Lithuanian -aitis, -(i)onis, (i)ünas, -uitis and Slavic -evič, -ovič, with diminutive suffixes and 2) anthroponyms without such suffixes (about this classification, read more in MACIEJAUSKIENE், 1993: 40; RAGAUSKAITE், 2018a: 104-106; 2019a: 182). Earlier studies into Lithuanian historical personal names of the 17th century had almost no mention of the use of the suffix -uitis. However, it was believed that the suffix -uitis was considered a patronymic suffix. This conclusion stems from the abundance of personal names with the suffix -uitis from Gruzdžiai parish of the 17th century as well as their detailed anthroponymic analysis (MACIEJAUSKIENE், 2010: 820). Among the studied personal names of patronymic origin that were particular to residents of Joniškis of the 16th - 17th centuries, derivatives of the suffix -uitis also comprised as many as $14.60 \%$ (RAGAUSKAITE், 2019a: 187, 194). 
Onomástica Desde América Latina, n.3, v.2, janeiro - junho, 2021, p. 2 - 29. ISSN 2675-2719 https://doi.org/10.48075/odal.v0i0.25781

As stated, only historical anthroponyms, which have not yet become hereditary personal names (surnames), could be broken up into a non-divisible structure (ZINKEVIČIUS, 1977: 238242; MACIEJAUSKIENĖ, 1997: 101-102; ŽEMIENE், 1999: 224-225; 2002: 11-14; 2017: 168; RAGAUSKAITE், 2005: 99-100; 2018a: 105-106; 2019a: 183; SINKEVIČIŪTĖ, 2018a: 120124; 2018b: 304-320). The analysed anthroponyms were reconstructed from patronyms and nonpatronymic personal names. The reconstructed forms of anthroponyms were marked by an asterisk and presented in alphabetical order. Then, personal names were listed (according to the alphabet of their forms), on the basis of which the form of the anthroponym was recreated. In some cases, several theoretically possible variants were left. These forms were compared to contemporary Lithuanian surnames. Failure to identify an exact equivalent in the contemporary onomastics resulted in the presentation of several more characteristic personal names with the respective root. In the spoken language, Lithuanians could have used the Lithuanian patronymic suffixes -aitis, énas, -onis, -ūnas (MACIEJAUSKIENĖ, 1994: 25-30; RAGAUSKAITE், 1999: 148-149; 2018b: 59). This is clearly demonstrated by the oldest inscriptions dated 1599-1600 of the parish register of baptism of Joniškis.

For the period 1599-1600, parish register of baptism of Joniškis contains 290 personal names of Joniškis villagers. Out of these, 274 (or $94.5 \%$ ) were attributed to the first group, which was mentioned earlier. This group was much larger. Anthroponyms are listed below by the productivity of their suffixes: 
Onomástica Desde América Latina, n.3, v.2, janeiro - junho, 2021, p. 2 - 29. ISSN 2675-2719

https://doi.org/10.48075/odal.v0i0.25781

-aitis (written as: -aitis, -aytis, -ä̈tis, -atis) - 182 (62.78 \%) personal names:

*Bagdonaitis : 1600 Bagdonaitis LBEPJ 14. Comp. curt. sur. Bagdonaitis (LPŽ ${ }^{14}$ I 153); *B a p kūna it is: 1600 Bapkunaitis LBEPJ 12v. Regarding bapkūn- comp. curt. sur. Bapkáuskas (LPŽ I 189); *Bučra it is: 1600 Buczraytis LBEPJ 18. Such surname does not function in the present-day onomastics; *Č ep a it is : 1600 Czepaitis LBEPJ 11v. Comp. curt. sur. Čepáitis (LPŽ I 410); *Dapkaitis: 1599 Dapkaitis LBEPJ 2. Comp. curt. sur. Dapkáitis (LPŽ I 459); *Dirmaitaitis: 1600 Dirmaitaitis LBEPJ 11v. Regarding dirmait- comp. curt. sur. Dirmáuskas, Dirmãvičius, Dirmeikà, Dirmeĩkis (LPŽ I 503); *Drobaitis: 1600 Drobaitis LBEPJ 14v. Regarding drob- comp. curt. sur. Dróba, Drobãvičius, Drobẽlis, Drobelis (LPŽ I 527); *El e kn a it i s : 1600 Eleknä̈tis LBEPJ 10v. Regarding elekn- comp. curt. sur. Eleknà (LPŽ I 571); *Endrūnaitis: 1599 Endrunatis LBEPJ 2v. Regarding endrūn- comp. curt. sur. Endrūnas (LPŽ I 577); *Endruškaitis: 1600 Endrufzkaitis LBEPJ 15. Comp. curt. sur. Endruškaitis (LPŽ I 577); *G e n a i t i s : 1600 Gienaitis LBEPJ 10. Comp. curt. sur. Genáitis (LPŽ I 650); *Ge ro na it is : 1600 Geronaitis LBEPJ 14v. Comp. curt. sur. Geronáitis (LPŽ I 658); *Giedraitis : 1599 Godraićia gen. sg. LBEPJ 2. Comp. curt. sur. Giedráitis (LPŽ I 664); *Gricaitis : 1599 Grycaycia gen. sg. LBEPJ 4v; 1599 Grycaitis LBEPJ 4v. Regarding griccomp. curt. sur. Grìcas, Gricẽvičius, Gricỹs, Griciùkas, Grìcius (LPŽ I 708, 709); *Griciūnaitis : 1599 Grycunaicia gen. sg. LBEPJ 3v. Regarding griciūn- comp. curt. sur. Griciūnas (LPŽ I 709); *G r ig a it is : 1600 Grigaitis LBEPJ 15v. Comp. curt. sur. Grigáitis (LPŽ I 710); *G riga la it is : 1600 Grigałaytis LBEPJ 16v. Comp. curt. sur. Grigaláitis (LPŽ I 711); *Ja s a i ti s : 1600 Jasaytis LBEPJ 10v. Comp. curt. sur. Jasáitis (LPŽ I 813); *Je ka it is : 1600

\footnotetext{
${ }^{14}$ All current surnames provided in the article are registered (LPŽ I, II - Maciejauskienè, V.; Razmukaite, M. \& Vanagas, A. (Ed.) (1985-1989). Lietuviu pavardžiu žodynas (Vol. 1-2). Vilnius: Mokslas).
} 
Onomástica Desde América Latina, n.3, v.2, janeiro - junho, 2021, p. 2 - 29. ISSN 2675-2719

https://doi.org/10.48075/odal.v0i0.25781

Iekaitis LBEPJ 13. Regarding jek- comp. curt. sur. Jekelis, Jekẽvičius (LPŽ I 827); *Je na it is : 1600 Jenaitis LBEPJ 19. Regarding jen- comp. curt. sur. Jenãvičius, Jenẽvičius, Jeniùlis (LPŽ I 828, 829); *Jokūbaitis: 1599 Jokubaycia gen. sg. LBEPJ 3v. Comp. curt. sur. Jókūbaitis, Jokūbáitis (LPŽ I 839); *Jo na it is : 1600 Janaytis LBEPJ 17v; 1600 Jonaicia gen. sg. LBEPJ 15v; 1599 Jonaitis LBEPJ 15; 1600 Jonaytis LBEPJ 11; 1599 Jonaÿtis LBEPJ 4v. Comp. curt. sur. Jonáitis (LPŽ I 841); *Jo n uša it i s : 1600 Janufzaitis LBEPJ 14; 1600 Jonufzaycia gen. sg. LBEPJ 4; 1600 Jonuszaytis LBEPJ 16v. Comp. curt. sur. Jõnušaitis, Jonušáitis (LPŽ I 844); *Jonuškaitis: 1600 Januszkaitis LBEPJ 9; 1600 Jonufzkaitis LBEPJ 4; 1600 Jonuszkaitis LBEPJ 8v. Regarding jonušk- comp. curt. sur. Jonuškà, Jonuškáitis, Jonuškẽvičius, Jonùškis (LPŽ I 844); *Juda it is : 1600 Judaytis LBEPJ 17v. Regarding jud- comp. curt. sur. Jùdas, Judèckis, Judeikà, Judeĩkis (LPŽ I 850, 851); *Judeikaitis: 1600 Judeikaitis LBEPJ 10. Regarding judeik- comp. curt. sur. Judeikà, Judeîkis (LPŽ I 850, 851); *J u ka it i s : 1600 Jukaitis LBEPJ 16. Regarding juk- comp. curt. sur. Jukãvičius, Jùkelis, Jukevičius (LPŽ I 852); *Ju kiš k a i ti s : 1600 Jukiszkaitis LBEPJ 15. Such surname does not function in the present-day onomastics; *Jukna it is : 1600 Juknaitis LBEPJ 8v. Comp. curt. sur. Juknáitis (LPŽ I 852); *J u lma i ti s : 1600 Jutmaitis LBEPJ 7v. Such surname does not function in the present-day onomastics; *Jurdo s a iti s : 1600 Jurdosaitis LBEPJ 10. Regarding jurd- comp. curt. sur. Jurdỹs (LPŽ I 864); *Jurgaitis: 1600 Jurgaitis LBEPJ 8v; 1600 Jurgaytis LBEPJ 17. Comp. curt. sur. Jùrgaitis, Jurgáitis, Jurgaitis (LPŽ I 866); *Ku ti n a it is : 1600 Kutinaitis LBEPJ 12v. Regarding kutin- comp. curt. sur. Kùtinas, Kutìnskas (LPŽ I 1141); *La u c a it i s : 1599 Laucaitis LBEPJ 1v. Comp. curt. sur. Laucáitis (LPŽ II 29); *La u ryna it is : 1600 Laurinaicia gen. sg. LBEPJ 15; 1600 Eaurinaytis LBEPJ 17; 1600 Lawrinaitis LBEPJ 9. Comp. curt. sur. Laurynáitis (LPŽ II 35); *Lig e ikaitis : 1599 Ligiekaytis LBEPJ 4v. Regarding ligeik- comp. curt. sur. Ligeikà, Ligeika, 
Onomástica Desde América Latina, n.3, v.2, janeiro - junho, 2021, p. 2 - 29. ISSN 2675-2719 https://doi.org/10.48075/odal.v0i0.25781

Ligeîkis (LPŽ II 79); *Linkaitis: 1600 Linkaitis LBEPJ 11v; 1600 Linkaitif LBEPJ 10v. Regarding link- comp. curt. sur. Lìnka, Linkà, Linkas, Linkẽlis (LPŽ II 86); *Lo c a it is : 1600 Łocaitis LBEPJ 14v. Comp. curt. sur. Locáitis (LPŽ II 104); *Lukošaitis : 1600 Eukoßaitis LBEPJ 8. Comp. curt. sur. Lukošáitis (LPŽ II 114); *Ma c a it i s : 1600 Macaÿcia gen. sg. LBEPJ 11; 1600 Macaitis LBEPJ 3; 1600 Macaytis LBEPJ 12; LBEPJ 17. Comp. curt. sur. Macáitis (LPŽ II 121); *Maciukaitis: 1600 Maciukaitis LBEPJ 13. Regarding maciuk- comp. curt. sur. Maciùkas (LPŽ II 124); *Mač i uka it is : 1599 Maćiukaicia gen. sg. LBEPJ 2. Comp. curt. sur. Mačiukáitis (LPŽ II 129); *Mantjurgaitis: 1599 Mantiurgaycia gen. sg. LBEPJ 2v. Such surname does not function in the present-day onomastics; *Martinaitis: 1599 Martinaitis LBEPJ 3v; 1600 LBEPJ 7v; 1600 Martinä̈tis LBEPJ 17v. Comp. curt. sur. Martináitis (LPŽ II 167); *Matulaitis: 1600 Matułaitis LBEPJ 9v. Comp. curt. sur. Matuláitis (LPŽ II 183); *M i c a i t is : 1600 Micaitis LBEPJ 15. Comp. curt. sur. Micáitis (LPŽ II 15); *Mic k a it i s : 1600 Mickaytis LBEPJ 11. Comp. curt. sur. Mickáitis (LPŽ II 218-219); *Mikaitis : 1599 Mikaitis LBEPJ 5; 1600 LBEPJ 11. Comp. curt. sur. Mikáitis (LPŽ II 225); *Mikutaitis: 1600 Mikutaitis LBEPJ 12; 1600 Mikutä̈tis LBEPJ 16v. Comp. curt. sur. Mikutaitis, Mikutáitis (LPŽ II 236); *Mockaitis : 1600 Mockaitis LBEPJ 17. Comp. curt. sur. Mockáitis (LPŽ II 262); *Mo ti eja it is : 1600 Moteiaycia gen. sg. LBEPJ 16v. Comp. curt. sur. Motiejáitis (LPŽ II 274); *Moz ūra it is : 1600 Mozuraitis LBEPJ 10; 1600 LBEPJ 12. Comp. curt. sur. Mozūráitis (LPŽ II 276); *Mu ra it i s : 1600 Muraitis LBEPJ 8. Regarding mur-comp. curt. sur. Mùralis, Mùralius, Muréika (LPŽ II 282, 283); *Nu ta it i s 1599 Nutaicia gen. sg. LBEPJ 3v. Regarding nut- comp. curt. sur. Nùtautas (LPŽ II 341); *P e c i u l a it is : 1600 Pecułaitis LBEPJ 11v. Regarding peciulcomp. curt. sur. Peciulẽvičius, Peciulis (LPŽ II 418); *Pe ki la it is : 1600 Pekiłaytis LBEPJ 16. Regarding pek- comp. curt. sur. Pekas, Pekỹs (LPŽ II 16); *P e tra it is : 1599 Patraitis LBEPJ 4; 
Onomástica Desde América Latina, n.3, v.2, janeiro - junho, 2021, p. 2 - 29. ISSN 2675-2719 https://doi.org/10.48075/odal.v0i0.25781

1599 Petraitis LBEPJ 5; 1600 LBEPJ 6v; 1600 Petraytis LBEPJ 16v; 1600 Petraÿtis LBEPJ 17v. Comp. curt. sur. Petráitis (LPŽ II 437); *Petrikaitis : 1599 Petrikaićia gen. sg. LBEPJ 1v; 1600 Petrikaitis LBEPJ 8v, 12v. Comp. curt. sur. Petrikáitis (LPŽ II 440); *P e tro š a it is : 1599 Petroszaicia gen. sg. LBEPJ 4. Comp. curt. sur. Petrošáitis (LPŽ II 442); *P i kt i na i ti s : 1600 Piktinaytis LBEPJ 15v. Regarding pikt- comp. curt. sur. Piktẽlis, Piktis, Piktỹs, Piktuĩzis, Piktužis (LPŽ II 453); *Povilaitis: 1600 Powełaitis LBEPJ 8v; 1600 Powiłaitis LBEPJ 9v; 1600 Powiłaytis LBEPJ 7v, 17v. Comp. curt. sur. Póvilaitis, Poviláitis, Povilaitis (LPŽ II 496-497); *Pūslaitis: 1599 Puzłaycia gen. sg. LBEPJ 3v. Comp. curt. sur. Pūsláitis (LPŽ II 540); *Ramonaitis: 1600 Ramonaitis LBEPJ 8v. Comp. curt. sur. Ramonáitis (LPŽ II 570); *R a to ša it is : 1599 Ratoßaitia gen. sg. LBEPJ 1. Such surname does not function in the presentday onomastics; *Ro ma na it is : 1600 Romanaitis LBEPJ 10. Comp. curt. sur. Romanaitis (LPŽ II 619); *Staka it is : 1600 Stakaycia gen. sg. LBEPJ 17v; 1600 Stakaytis LBEPJ 17v. Comp. curt. sur. Stakáitis (LPŽ II 794); *S ta n a it i s : 1599 Stanaycia gen. sg. LBEPJ 2v; 1599 Stanaitis LBEPJ 3; 1600 LBEPJ 13. Comp. curt. sur. Stanáitis (LPŽ II 798); *Sta ni ula it is : 1600 Staniułaytis LBEPJ 11v. Comp. curt. sur. Staniuláitis (LPŽ II 802); *S te n i u ka itis: 1600 Steniukaycia gen. sg. LBEPJ 11v. Regarding steniuk- comp. curt. sur. Steniukýnas (LPŽ II 818); * ̌́ ima it is : 1600 Szymaitis LBEPJ 3. Comp. curt. sur. Šimáitis (LPŽ II 928); * ̌́ve paitis: 1600 Szwepaitis LBEPJ 14v. Such surname does not function in the present-day onomastics; * ̌̌v vit a it is : 1599 Świtaicia gen. sg. LBEPJ 2. Regarding švit- comp. curt. sur. Švitinis, Švitìnskas (LPŽ II 1006); *Ta mo ša i ti s : 1599 Tamoszaitis LBEPJ 4. Comp. curt. sur. Tamošáitis (LPŽ II 1016); *Ta mu la it is : 1599 Tamułaitim LBEPJ 10. Comp. curt. sur. Tamuláitis (LPŽ II 1017); *Tüba it is : 1600 Thubaitis LBEPJ 7v. Comp. curt. sur. Tübaitis (LPŽ II 1076); *Va li na it is : 1599 Walinaycia gen. sg. LBEPJ 4. Comp. curt. sur. Vàlinaitis (LPŽ II 1151-1152); 
Onomástica Desde América Latina, n.3, v.2, janeiro - junho, 2021, p. 2 - 29. ISSN 2675-2719 https://doi.org/10.48075/odal.v0i0.25781

*Vali ula itis : 1600 Valułaitis LBEPJ 15. Regarding valiul- comp. curt. sur. Valiùlis (LPŽ II 1153-1154); *Valutaitis : 1600 Valutaycia gen. sg. LBEPJ 17. Regarding valut- comp. curt. sur. Valùtis (LPŽ II 1158); *Verbušaitis: 1600 Verbuszaitis LBEPJ 15. Comp. curt. sur. Verbušáitis (LPŽ II 15); *Vikutaitis : 1600 Wykutaytis 16v. Regarding vikut- comp. curt. sur. Vikùtis (LPŽ II 1209); *Z a s a it is : 1599 Zajsajcia gen. sg. LBEPJ 4v. Regarding zas- comp. curt. sur. Zãsas (LPŽ II 4v); *Ža ra ga it i s : 1599 Zaragaicia gen. sg. LBEPJ 3. Such surname does not function in the present-day onomastics; *Žlegaitis: 1600 Zlegaitis LBEPJ 12. Such surname does not function in the present-day onomastics.

-(i)ünas (written as -(i)uns) - 53 (18.28 \%) anthroponyms: *Bartkūnas : 1600 Bartkuna gen. sg. LBEPJ 4. Comp. curt. sur. Bartkūnas (LPŽ I 204); *Be tk ū na s : 1599 Betkuna gen. sg. LBEPJ 4. Regarding betk- comp. curt. sur. Betkáuskas, Bètkè, Bètkis, Bẽtkus (LPŽ I 245); *Bliūdžiūnas: 1600 Btudziuns LBEPJ 11v. Comp. curt. sur. Bliudžiūnas (LPŽ I 278); *Dapkūnas: 1600 Dapkuns LBEPJ 12v. Comp. curt. sur. Dapkūnas (LPŽ I 459-460); *Griciūnas: 1600 Grićiuns LBEPJ 9v. Comp. curt. sur. Griciūnas (LPŽ I 709); *Grigali ünas: 1600 Grigaluna gen. sg. LBEPJ 8; 1600 Grigaluns LBEPJ 17v. Comp. curt. sur. Grigaliūnas (LPŽ I 711); *Grini ūnas: 1600 Griniuns LBEPJ 14v. Comp. curt. sur. Griniūnas (LPŽ I 14v); *Iz dūn as : 1600 Izduns LBEPJ 10. Regarding izdūn- comp. curt. sur. Izdūnis (LPŽ I 778); *Ja un ni ūnas : 1600 Jawnuns LBEPJ 9. Regarding jaun- comp. curt. sur. Jaunas, Jauneikà, Jaũniškis, Jauniškis, Jaũnius (LPŽ I 824); *Jenkūnnas: 1600 Jenkuna gen. sg. LBEPJ 14. Regarding jenk- comp. curt. sur. Jenkaũskas, Jenkelis, Jenkẽvičius, Jénkis, Jenkys, Jeñkus (LPŽ I 14); *Jo nkūn as : 1600 Jonkuna gen. sg. LBEPJ 14v. Comp. curt. sur. Jonkūnas (LPŽ I 843); *Ju c i ū na s: 1600 Jućiuna gen. sg. LBEPJ 9. Comp. curt. sur. Juciūnas (LPŽ I 850); *Kiemenčiūnas: 1600 Kiemienciuns LBEPJ 12v. Such surname does not function in the 
Onomástica Desde América Latina, n.3, v.2, janeiro - junho, 2021, p. 2 - 29. ISSN 2675-2719 https://doi.org/10.48075/odal.v0i0.25781

present-day onomastics; *Kr unko ni ünas : 1600 Krunnkoniuna gen. sg. LBEPJ 10v. Regarding krunk- comp. curt. sur. Krunkáitis, Krunkáuskas (LPŽ I 1091); *La u ci ūn as : 1599 Łauciuns LBEPJ 2v; 1600 Łaucuns LBEPJ 7. Comp. curt. sur. Lauciūnas (LPŽ II 17); * Mi ka lojū na s : 1600 Mikatoiuns LBEPJ 11; 1600 Mikatoiun LBEPJ 9v. Comp. curt. sur. Mikalojūnas (LPŽ II 227); *Morkūnas: 1600 Morkuns LBEPJ 15v. Comp. curt. sur. Morkūnas (LPŽ II 271); *Motiejūnas: 1600 Moteiuns LBEPJ 16v. Comp. curt. sur. Motiejūnas (LPŽ II 274); *Petroši ūnas: 1600 Petrośiuns LBEPJ 10v; 1600 Petroßiuns LBEPJ 15v. Comp. curt. sur. Petrošiūnas (LPŽ II 442); *Pociūnas : 1600 Pociuns LBEPJ 21. Comp. curt. sur. Pociūnas (LPŽ II 483); *Sarg ūna s : 1600 Sarguna gen. sg. LBEPJ 12. Comp. curt. sur. Sargūnas (LPŽ II 678); *Satkūnas : 1600 Sotkuna gen. sg. LBEPJ 16. Comp. curt. sur. Satkūnas (LPŽ II 16); *Stankūnas: 1600 Stankuns LBEPJ 8v. Comp. curt. sur. Stankūnas (LPŽ II 804); *St a s i ū nas : 1600 Stafiuns LBEPJ 17. Comp. curt. sur. Stasiūnas (LPŽ II 810); *Š i d a g ū na s : 1600 Sidaguns LBEPJ 7v. Regarding šidag- comp. curt. sur. Šidagis (LPŽ II 921-922); *Šileikūnas : 1600 Siteÿkuns LBEPJ 16v. Regarding šileik- comp. curt. sur. Šiléika, Šileikà, Šileikauskas, Šileîkis (LPŽ II 925); *Tū bi ūnas : 1599 Tubiuna gen. sg. LBEPJ 1v. Regarding tūb- comp. curt. sur. Tūbà, Tūbaitis, Tūbẽlis, Tūbìnis (LPŽ II 1076, 1077); *Vale nči ì nas : 1600 Valenciuns LBEPJ 16. Comp. curt. sur. Valenčiūnas (LPŽ II 16); *Vili ū na s : 1599 Viluna gen. sg. LBEPJ 4; 1600 Wiluns LBEPJ 15v. Comp. curt. sur. Viliūnas (LPŽ II 1216); *Zari ūnas : 1599 Zariun LBEPJ 5v - 1599 Zarun LBEPJ 4v. Regarding zar- comp. curt. sur. Zãras, Zaráuskas (LPŽ II 1274).

-uitis (written as: -(i)uitis, -uić, -uytis, -uÿtis)-28 (9.68 \%) personal names: *B a u k uiti s : 1600 Baukuitis LBEPJ 11v. Regarding bauk- comp. curt. sur. Baũkus, Baukẽvičius, Baukỹs, Baũkus (LPŽ I 214); *B i k i e k u it i s : 1600 Bikiekuitis LBEPJ 7v. Such surname does not function 
Onomástica Desde América Latina, n.3, v.2, janeiro - junho, 2021, p. 2 - 29. ISSN 2675-2719 https://doi.org/10.48075/odal.v0i0.25781

in the present-day onomastics; *B u ilu it is : 1600 Buytuitis LBEPJ 14v. Regarding buil- comp. curt. sur. Buĩlis, Builỹs (LPŽ I 335); *D ro b u it i s : 1600 Drobuytis LBEPJ 17v. Regarding drobcomp. curt. sur. Dróba, Drobãvičius, Drobẽlis, Drobelis (LPŽ I 527); *Gebruitis: 1600 Gebruitis LBEPJ 9. Regarding gebr- comp. curt. sur. Gebráuskas (LPŽ I 637); *J e k u i t i s : 1600 Jekuitis LBEPJ 12. Regarding jek- comp. curt. sur. Jekelis, Jekẽvičius (LPŽ I 827); *J u rk u i t is : 1600 Jurkuitis LBEPJ 17. Regarding jurk- comp. curt. sur. Jurkáitis (LPŽ I 868-869); *Ku ke luitis : 1600 Kukietuyćia gen. sg. LBEPJ 16v. Regarding kuk- comp. curt. sur. Kukẽnis, Kukenỹs, Kukẽvičius, Kùkis (LPŽ I 1108); *Lenipuitis: 1600 Lenipuitis LBEPJ 15v. Such surname does not function in the present-day onomastics; *Linuitis: 1600 Linuicia gen. sg. LBEPJ 14. Regarding lin- comp. curt. sur. Linas, Lináuskas, Liniónis, Linónis (LPŽ II 87); *Paukštuluitis : 1599 Pauxtułuitis LBEPJ 1. Regarding paukštul- comp. curt. sur. Paukšta, Paukštáitis, Paũkštè, Paukštẽlis, Paũkštis, Paukštỹs (LPŽ II 407, 408); *Pažuitis: 1599 Pazuićia gen. sg. LBEPJ 1v. Such surname does not function in the present-day onomastics; *Petrikuitis : 1600 Petrikuitis LBEPJ 16v. Regarding petrik- comp. curt. sur. Petrikáitis (LPŽ II 440); *Pirtinikuitis: 1600 Pirtinikuitis LBEPJ 9v. Regarding pirtin- comp. curt. sur. Pìrtinas (LPŽ II 462-463); *Sp ir a ku iti s: 1600 Spirakuytis LBEPJ 11. Such surname does not function in the present-day onomastics; *Sruoguitis: 1600 Sroguić LBEPJ 7v. Regarding sruog- comp. curt. sur. Srúoga, Sruogè, Sruoginis, Sruoginỹs, Sruogis, Srúogius (LPŽ II 789); *Ša ri u it is : 1599 Szariuitis LBEPJ 2v. Regarding šar- comp. curt. sur. Šaráitis, Šãras (LPŽ II 888, 889); *Šiulpikuitis: 1600 Sziutpikuitis LBEPJ 8v. Such surname does not function in the present-day onomastics; *Šo puitis : 1600 Szopuitis LBEPJ 16. Regarding šop- comp. curt. sur. Šópa, Šopaila, Šópas, Šópis, Šopỹs (LPŽ II 16); *Tydikuitis: 1600 Tydykuitis LBEPJ 12. Regarding tydik- comp. curt. sur. Tydikas (LPŽ II 1042); *Va i žg u i ti s : 1600 Wayzguitis LBEPJ 
Onomástica Desde América Latina, n.3, v.2, janeiro - junho, 2021, p. 2 - 29. ISSN 2675-2719 https://doi.org/10.48075/odal.v0i0.25781

17v. Regarding vaižg- comp. curt. sur. Vaižgaũskas (LPŽ II 1143); *Velykuitis: 1600 Weliku[icia] gen. sg. LBEPJ 14v. Regarding velyk- comp. curt. sur. Velyka, Velykenas, Velỹkis (LPŽ II 1184); *Zaruitis : 1600 Zarü̈tis LBEPJ 16. Regarding zar- comp. curt. sur. Zãras, Zaráuskas (LPŽ II 1274).

-onis (-ons) - 2 (0.68 \%) anthroponyms: *Grubonis: 1600 Grubons LBEPJ 13v. Regarding grub- comp. curt. sur. Grubà, Grùbas, Grubãvičius, Grubè, Grùbis, Grubỹs (LPŽ I 726); *Si da u g o ni a gen. sg. LBEPJ 10v. Regarding sidaug- comp. curt. sur. Sìdauga (LPŽ II 709).

-(i)evič (written as -(i)ewicz) - 1 (0. 34 \%) personal name: 1599 Januszkiewicz LBEPJ 1. -ovič (written as -owicz) - 1 (0.34 \%) anthroponym: 1600 Woićiechowicz LBEPJ 9v.

At least 7 personal names of this group had diminutive suffixes. This type of suffixes may also have a patronymic meaning (ZINKEVIČIUS, 1977: 238; MACIEJAUSKIENĖ, 1991: 227; 1993: 43; RAGAUSKAITE், 2005: 100-101). Anthroponyms are listed by the frequency of derivatives:

-(i)ulis (written as -(i)ulis) - 4 (1.38 \%) personal names: *Je n i u li s : 1600 Jeniulis LBEPJ 13. Comp. curt. sur. Jeniùlis (LPŽ I 13); *Ma tu li s : 1600 Matulis LBEPJ 15. Comp. curt. sur. Matùlis (LPŽ II 184); *S t a n i u l i s : 1600 Staniulis LBEPJ 9v, 14. Comp. curt. sur. Staniùlis (LPŽ II 802).

-ele (-el) - 1 (0,34 \%) anthroponym: *Barzdelè : 1599 Barzdeły gen. sg. LBEPJ 5v. Dél barzd- plg. db. pvd. Barzdẽlis (LPŽ I 207). 
Onomástica Desde América Latina, n.3, v.2, janeiro - junho, 2021, p. 2 - 29. ISSN 2675-2719

https://doi.org/10.48075/odal.v0i0.25781

-elis (-elis) - $1(0,34 \%)$ personal name: *Tvabelis : 1600 Twabelis LBEPJ 12. Such surname does not function in the present-day onomastics.

-utis (-uc) - 1 (0,34 \%) anthroponym: *Mackutis : 1599 Mackucia gen. sg. LBEPJ 3. Dèl mack- plg. db. pvd. Mãckus (LPŽ II 126).

Another group of personal names inscribed in the source were of non-patronymic origin. They amounted to as few as $16(5.5 \%)$. Anthroponyms of this group were compound words and ending derivatives. From 16 personal names recorded in the source, 16 proper name forms were reconstructed with formants particular to Lithuanian formation. Namely: *B ajo r a s : 1600 Baiors LBEPJ 8. Comp. curt. sur. Bajõras (LPŽ I 160); *Buvainis: 1600 Buwainis LBEPJ 11. Regarding buvain- comp. curt. n. Bùvainas (LVKŽ 106); *D rukis : 1600 Drukis LBEPJ 8. Such surname does not function in the present-day onomastics; *Ele čkas : 1600 Eleczkas LBEPJ 11. Such surname does not function in the present-day onomastics; *Ivoškis: 1599 Jwoszkia gen. sg. LBEPJ 3v-4. Comp. curt. sur. Ivõškis, Ivòškis (LPŽ I 778); *Jakštas : 1600 Jaxtas LBEPJ 12. Comp. curt. sur. Jãkštas (LPŽ I 789); * Ka i r i s : 1599 Kayris LBEPJ 5. Comp. curt. sur. Kaĩris (LPŽ I 888); *Klèsas: 1600 Klesas LBEPJ 12v. Comp. curt. sur. Klèsas (LPŽ I 1018); *Kremenčius: 1600 Kremenćius LBEPJ 18. Such surname does not function in the presentday onomastics; *Melnikas : 1600 Melniks LBEPJ 10. Comp. curt. sur. Mélnikas (LPŽ II 207); *Petrošius : 1600 Petrosius LBEPJ 8v. Comp. curt. sur. Petrõšius (LPŽ II 442); *Plukis: 1600 Ptukis LBEPJ 17. Comp. curt. sur. Plùkis (LPŽ II 481); *Skè ris: 1599 Skieris LBEPJ 5. Comp. curt. sur. Skèris, Skèrỹs (LPŽ II 5); *Užkuris : 1600 VszkurifLBEPJ 8v. Comp. curt. sur. Ǔ̌kuris, Užkurỹs (LPŽ II 1121); *Vaškus : 1600 Vaszkus LBEPJ 14. Comp. curt. sur. Vãškus (LPŽ II 1172); *Vidg in is : 1600 Witginis LBEPJ 17. Comp. curt. sur. Vidgìnis (LPŽ II 1203). 
Onomástica Desde América Latina, n.3, v.2, janeiro - junho, 2021, p. 2 - 29. ISSN 2675-2719 https://doi.org/10.48075/odal.v0i0.25781

Following the description on the structure of anthroponyms characteristic to Joniškis parish villagers of the 16th century, 166 reconstructed forms were compared to contemporary surnames of Lithuanians. It became apparent that 87 (or $52.41 \%$ of all the reconstructed) are still used at present. A portion, i.e. 60 (or $36.15 \%$ ), had equivalents of contemporary surnames of the same origin yet different formation. The remaining $19(11.44 \%)$ recreated personal names had no equivalents in the contemporary onomastics. They could have emerged due to irregularities of inscriptions resulting from the lack of care on the part of scribes, their incompetence or other extralinguistic circumstances (the interplay between Lithuanian, Polish and Latin languages).

\section{Results}

(1) 290 anthroponyms (second members of the binary naming model) characteristic to villagers of 1599-1600 were collected from the parish register of baptism of Joniškis. First members of these binary naming instances were Christian names. Various forms of names were used (traditional, hipocoristics, and diminutive forms of the hypocoristics) (Adamus, Gregorius, Stanislaus; Genis, Macis, Stas; Geniulis, Jonelis, Staniulis).

(2) The 290 analysed personal names were classified into two groups: 1) anthroponyms with patronymic suffixes, namely, Lithuanian -aitis, -(i)onis, -(i)ünas, -uitis and Slavic-evič, $\quad$-ovič, with diminutive suffixes -ele, -elis, -(i)ulis, -utis and 2) anthroponyms without such suffixes.

(3) The first group was much larger. It comprised 274 (or 94.5\%) of all personal names. Among them, the most frequent were derivatives of patronymic suffixes -aitis (182 or $62.78 \%$ ) and - 
Onomástica Desde América Latina, n.3, v.2, janeiro - junho, 2021, p. 2 - 29. ISSN 2675-2719 https://doi.org/10.48075/odal.v0i0.25781

(i)ūnas (18.28\%) (Mozūraitis, Pūslaitis, Romanaitis; Griniūnas, Morkūnas, Pociūnas). The second group had $16(5.5 \%)$ anthroponyms (Bajoras, Ivoškis, Jakštas).

(4) The comparison of the forms of 166 reconstructed personal names with contemporary surnames of Lithuanians determined that $87(52.41 \%)$ were functional at present (Jeniulis, Petrikaitis, Lauciūnas). The smaller part, i.e. 60 (36.15\% of all recreated anthroponyms) had equivalents of contemporary surnames of the same origin yet different formation (Buvainis, Šopuitis, Valiulaitis). The remaining $19(11.44 \%)$ were not available in contemporary index of the surnames (Drukis, Kiemenčiūnas, Pažuitis).

\section{Appendices}


Onomástica Desde América Latina, n.3, v.2, janeiro - junho, 2021, p. 2 - 29. ISSN 2675-2719 https://doi.org/10.48075/odal.v0i0.25781

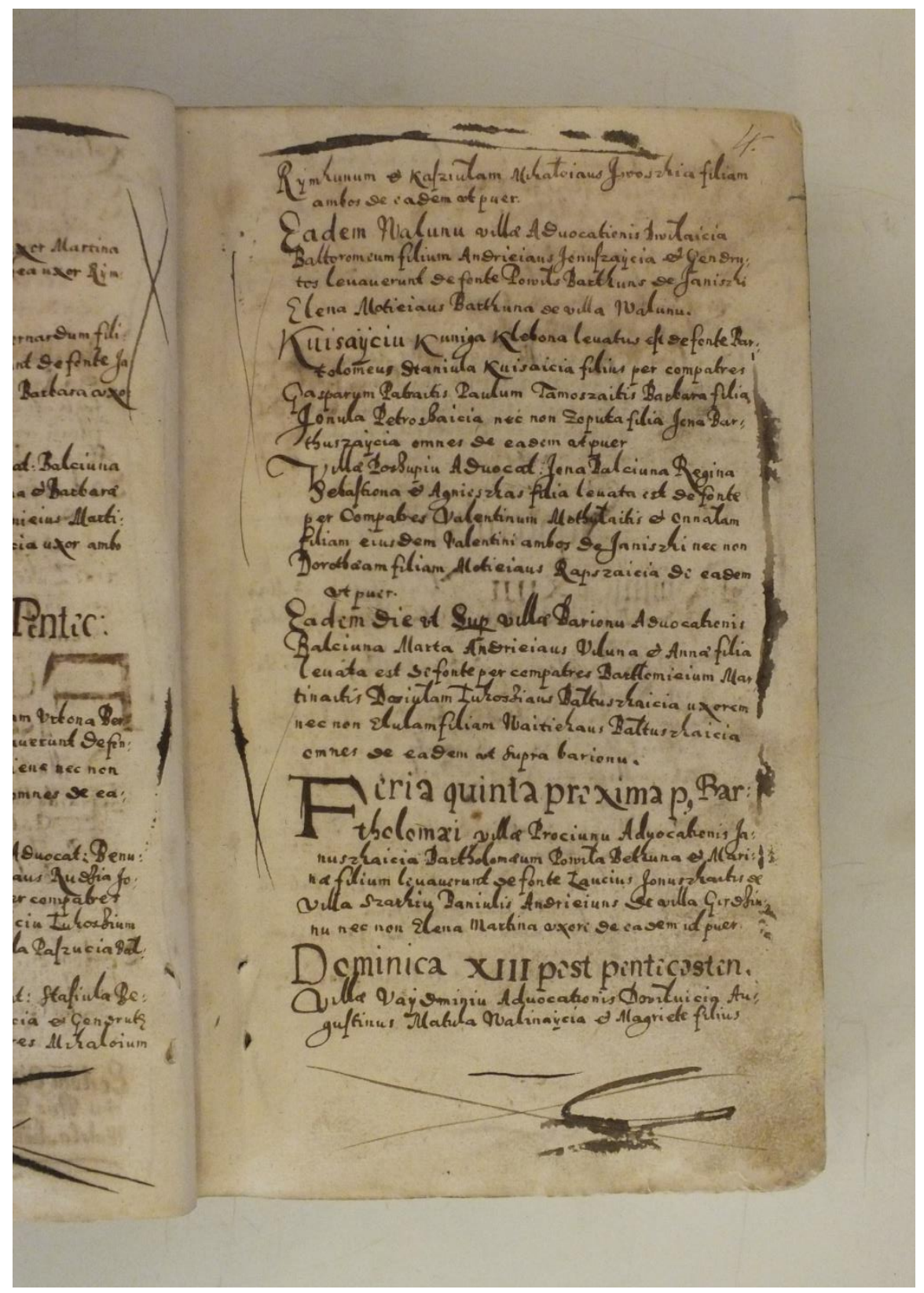

Figure 1. The Catholic parish in Joniškis, Register of baptism, 1599-1621, fragment dated 1599 (p. 4) 
Onomástica Desde América Latina, n.3, v.2, janeiro - junho, 2021, p. 2 - 29. ISSN 2675-2719 https://doi.org/10.48075/odal.v0i0.25781 
Onomástica Desde América Latina, n.3, v.2, janeiro - junho, 2021, p. 2 - 29. ISSN 2675-2719 https://doi.org/10.48075/odal.v0i0.25781

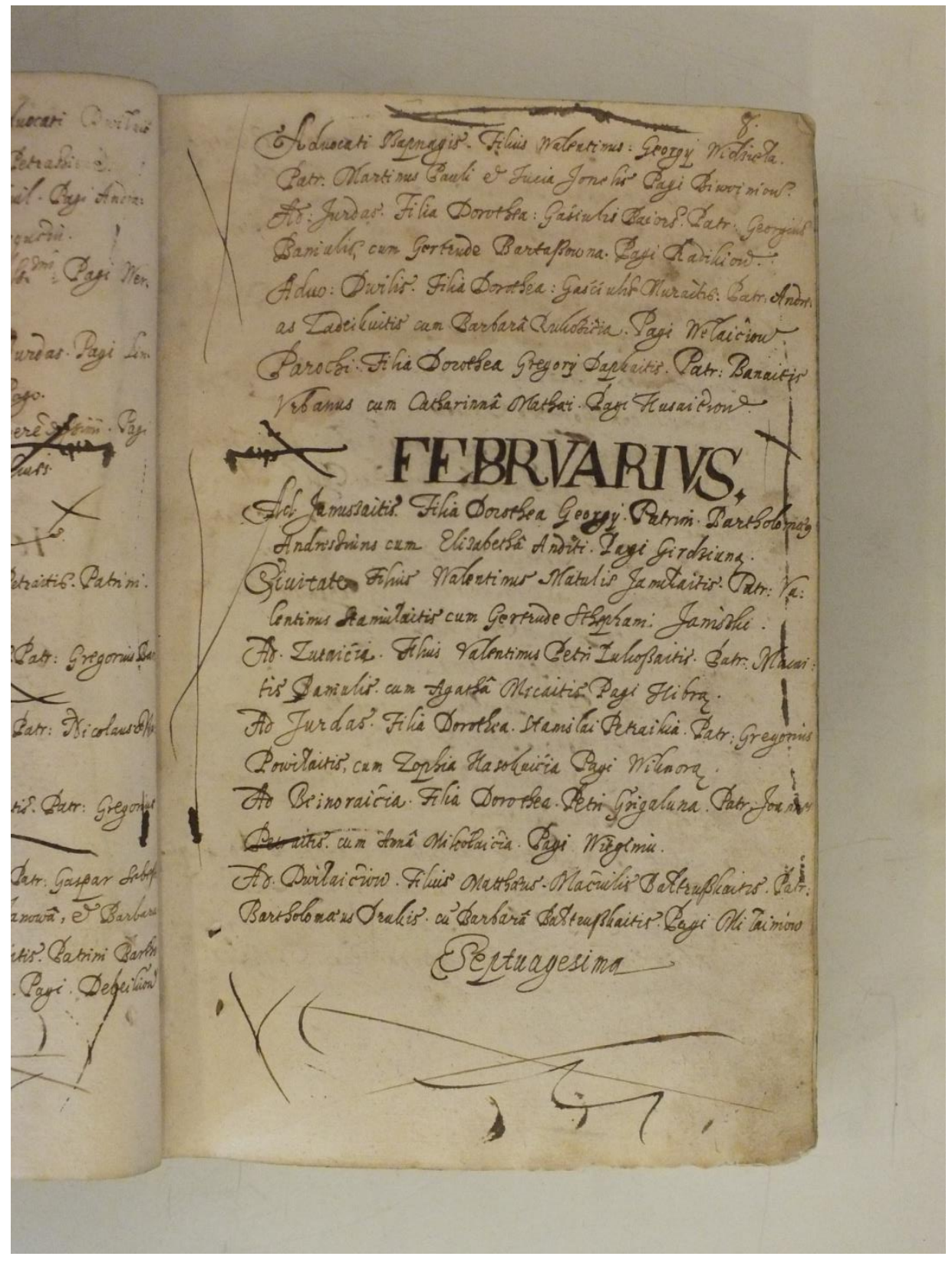

Figure 2. The Catholic parish in Joniškis, Register of baptism, 1599-1621, fragment dated 1600 (p. 8) 


\section{Archival source}

LBEPJ - Liber Baptisatoru[m] \| Ecc[lesi]ae Parochialis Jani=\|scensis ab Anno 1599. - \|

usq[ue] ad An[n]um || 1621. - [Catholic parish in Joniškis, Register of baptism, 1599-1621] Lietuvos valstybès istorijos archyvas [Office of the Chief Archivist of Lithuania], LVIA, f. 1196, ap. 1, b. 76 .

\section{Bibliography}

Ališauskas, V.; Jaszczołt T.; Jovaiša, L. \& Paknys, M. (2009). Lietuvos kataliku dvasininkai XIVXVI a.: Bažnyčios istorijos studijos (Vol. 2). Vilnius: Aidai.

Amaral, E. T. R; Seide, M. S. (2020a). Nomes próprios de pessoa: introdução à antroponímia brasileira. Blucher: São Paulo. https://openaccess.blucher.com.br/article-list/9786555500011$\underline{453 / \text { list\#undefined }}$

Garliauskas, V. (1998). Lietuviškos oikonimų lytys nelietuviškuose XVI-XIX a. šaltiniuose. Lietuviu kalbotyros klausimai, 40, 1998, 123-204.

Garliauskas, V. (2004). XVII a. Molètu bažnyčios krikšto ir santuokos metriku knyga. Vilnius: Mokslo ir enciklopedijų leidybos institutas.

LPŽ I - Maciejauskienė, V.; Razmukaite, M. \& Vanagas, A. (Ed.) (1985). Lietuviu pavardžiu žodynas (Vol. 1). Vilnius: Mokslas.

LPŽ II - Maciejauskienè, V.; Razmukaitė, M. \& Vanagas, A. (Ed.) (1989). Lietuviu pavardžiu žodynas (Vol. 2). Vilnius: Mokslas. 
Onomástica Desde América Latina, n.3, v.2, janeiro - junho, 2021, p. 2 - 29. ISSN 2675-2719

https://doi.org/10.48075/odal.v0i0.25781

LVKŽ - Kuzavinis, K., \& Savukynas, B. (1994). Lietuviu vardų kilmés žodynas. Vilnius: Mokslo ir enciklopedijų leidykla.

Maciejauskienè, V. (1991). Lietuviu pavardžiu susidarymas XIII-XVIII a. Vilnius: Mokslas.

Maciejauskienė, V. (1993). Kriaunų parapijos XVII-XVIII a. asmenvardžiai. Lietuviu kalbotyros

klausimai, 32: Baltu onomastikos tyrimai, 34-99.

Maciejauskienė, V. (1994). Lietuviu pavardès: raida ir kilmé (habilitacinis darbas). Vilnius:

Lietuvių kalbos institutas.

Maciejauskienė, V. (1997). Dėl lietuvių pavardžių (asmenvardžių klasifikavimo). Baltistica, 32(1), 101-109. DOI: 10.15388/baltistica.32.1.407

Maciejauskienè, V. (2010). Gruzdžių pavardès: istorija ir dabartis. In D. Šniukas (Ed.), Gruzdžiai (Vol. 2, pp. 818-844). Vilnius: Versmè.

Ragauskaitė, A. (1999). XVI a. II pusės Kauno miestiečiu lietuviškos kilmės asmenvardžiai. Acta Linguistica Lithuanica / Lietuviu kalbotyros klausimai, 41, 145-158.

Ragauskaite, A. (2000). XVII a. pirmosios pusės Kauno miestiečių asmenvardžių kilmè. Acta Linguistica Lithuanica / Lietuviu kalbotyros klausimai, 43, 93-110.

Ragauskaite, A. (2001). Kauno miestiečiu pavardžių formavimasis XVI-XVIII a. Acta Linguistica Lithuanica / Lietuviu kalbotyros klausimai, 45, 123-143.

Ragauskaitė, A. (2002). XVII-XVIII amžių kauniečių (vyrų) pavardžiu variantai. Acta Linguistica Lithuanica / Lietuviu kalbotyros klausimai, 47, 31-40.

Ragauskaite, A. (2003). XVI-XVIII a. kauniečiu moterų asmenvardžių daryba. Vārds un tā pētišanas aspekti: Rakstu krājums, 7, 82-92. 
Onomástica Desde América Latina, n.3, v.2, janeiro - junho, 2021, p. 2 - 29. ISSN 2675-2719 https://doi.org/10.48075/odal.v0i0.25781

Ragauskaitė, A. (2004). XVI-XVII a. joniškiečiu asmenvardžiai. In J. Švambarytė-Valužienė (Ed.), Istoriniai tekstai ir vietos kultūra (pp. 8-18). Šiauliai, Ryga: Lucilijus.

Ragauskaite, A. (2005). XVI-XVIII a. kauniečiu asmenvardžiai. Vilnius: Lietuvių kalbos instituto leidykla.

Ragauskaité, A. (2006). Vietovardinès kilmès asmenvardžiai ir prievardžiai XVI a. Kauno aktų knygose. Baltu filoloǵija, 15(1-2), 81-94.

Ragauskaite, A. (2008). Tendencies in the evolution of hereditary surnames of townspeople in Lithuania from sixteenth to eighteenth centuries. Zunamen / Surnames, 3, 29-37.

Ragauskaitè, A. (2009). Kada susidarè kauniečių pavardès? In A. Judžentis (Ed.), Kalba ir žmonès (pp. 226-232). Vilnius: Vilniaus universiteto leidykla.

Ragauskaitė, A. (2014a). Kauno moterų asmenvardžiai XVI-XVII a. lietuvių istorinès antroponimijos kontekste. Kauno istorijos metraštis, 14, 7-17.

Ragauskaite, A. (2014b). Lietuvoje sudarytas rankraštinis tikrinių vardų registras (apie 18401843). Archivum Lithuanicum, 16, 323-340.

Ragauskaite, A. (2015). Seniausios Lietuvoje 1599-1621 metų Joniškio krikšto metrikų knygos joniškiečių moterų asmenvardžiai lietuvių istorinès antroponimijos kontekste. Acta Linguistica Lithuanica, 73, 55-81.

Ragauskaitè, A. (2016a). Škotų pavardžių užrašymo tendencijos XVII-XVIII a. Kėdainių miesto istorijos šaltiniuose. Acta Linguistica Lithuanica, 75, 45-67. 
Onomástica Desde América Latina, n.3, v.2, janeiro - junho, 2021, p. 2 - 29. ISSN 2675-2719 https://doi.org/10.48075/odal.v0i0.25781

Ragauskaitè, A. (2016b). Germaniškos kilmės kėdainiečių antroponimai XVII-XVIII a. Kėdainių miesto istorijos šaltiniuose. Baltu filoloǵija, 25(2), 111-128.

Ragauskaitė, A. (2017b). Kėdainiečiu (lietuvių, lenkų) pavardžių formavimosi tendencijos XVIIXVIII amžiuje. Acta Linguistica Lithuanica, 77, 53-77.

Ragauskaitè, A. (2018a). XVII a. kèdainiečiu asmenvardžių darybos tendencijos. Baltu filologija, 27, 101-118. DOI: http://doi.org/10.22364/bf.27.04

Ragauskaitè, A. (2018b). XVII a. kėdainiečių lietuviškos kilmès asmenvardžiai. Acta Linguistica Lithuanica, 78, 53-75.

Ragauskaite, A. (2018c). The patterns of recording the names of German residents in Kèdainiai in the nineteenth century: Introductory remarks. Acta Baltico-Slavica, 42, 173-188. DOI: $10.11649 / \mathrm{abs} .2018 .009$

Ragauskaitė, A. (2019a). Joniškiečių vyrų asmenvardžių darybos tendencijos seniausioje Lietuvoje 1599-1621 m. Joniškio krikšto metrikų knygoje. Acta Linguistica Lithuanica, 80, 177194. DOI: https://doi.org/10.35321/all80-08

Ragauskaite, A. (2019b). Lietuviškos kilmès asmenvardžiai seniausioje Lietuvoje 1599-1621 m. Joniškio krikšto metrikų knygoje. Baltu filologija, 28(1), 153-168. DOI: http://doi.org/10.22364/bf.28.1.06

Seide, M. S.; Petrulione, L. (2018). Between Languages and Cultures: an Exploratory Comparative Study of Usage of Lithuanian and Brazilian Masculine Anthroponyms. Revista de estudos da Linguagem, 26, 1201-1226. 
Onomástica Desde América Latina, n.3, v.2, janeiro - junho, 2021, p. 2 - 29. ISSN 2675-2719

https://doi.org/10.48075/odal.v0i0.25781

http://www.periodicos.letras.ufmg.br/index.php/relin/article/view/12745

Seide, M. S.; Petrulionè, L. (2020). Formation and usage of hypocoristic forms in Brazilian, Portuguese and Lithuanian. Revista Alfa, 64(1), 1-27.

https://periodicos.fclar.unesp.br/alfa/article/view/11611/9330

Sinkevičiūtè, D. (2018a). Nežinomi Lietuvos Metrikos senieji lietuvių dvikamieniai asmenvardžiai. Baltistica, 53(1), 117-132. DOI: $\underline{\text { 10.15388/baltistica.53.1.2346 }}$

Sinkevičiūtė, D. (2018b). Nežinomi Lietuvos Metrikos dvikamieniai asmenvardžiai su pirmaisiais apeliatyviniais dèmenimis. Baltistica, 53(2), 303-325.

DOI: $\underline{10.15388 / \text { baltistica.53.2.2351 }}$

Zinkevičius, Z. (1977). Lietuvių antroponimika. Vilniaus lietuvių asmenvardžiai XVII a. pradžioje. Vilnius: Mokslas.

Žemienė, A. (1999). Ragainès apskrities XVIII a. pab. - XIX a. pr. vyrų pavardžių daryba. Acta Linguistica Lithuanica / Lietuviu kalbotyros klausimai, 41, 223-231.

Žemienè, A. (2002). XVIII-XIX a. Ragainès apskrities lietuvininku pavardžiu kilmé. Daktaro disertacijos santrauka. Vilnius: Vilniaus pedagoginis universitetas.

Žemienè, A. (2017). Mažosios Lietuvos asmenvardžiai (XVIII-XIX a.). Vilnius: Technika.

DOI: https://doi.org/10.20334/2017-041-M

Žilys, S. (2012). Bažnyčių metrikų knygos ir parapijiečių sąrašai Lietuvos mokslų akademijos Vrublevskių bibliotekos Rankraščių skyriuje: kilmė ir konfesiniai ypatumai. - In A. Pacevičius 
Onomástica Desde América Latina, n.3, v.2, janeiro - junho, 2021, p. 2 - 29. ISSN 2675-2719 https://doi.org/10.48075/odal.v0i0.25781

(Ed.), Atminties institucijų rinkiniai. Bibliotheca Lithuana (Vol. 2, pp. 123-154). Vilnius: Vilniaus universiteto leidykla. DOI: https://doi.org/10.15388/BiblLita.2012.2.15578 